\title{
Significance of a Novel Apolipoprotein E Variant, ApoE Osaka/ Kurashiki, in Lipoprotein Glomerulopathy
}

Lipoprotein glomerulopathy (LPG) is a unique kidney disease characterized by lipoprotein thrombi in the glomerulus ${ }^{1)}$. Type III hyperlipoproteinemia with a high apolipoprotein E (apoE) level in serum is a distinctive feature in the lipoprotein profile ${ }^{2)}$. Approximately 100 cases of LPG have been reported in the world since we published the first case in the American Journal of Kidney Diseases in $1989^{3)}$, and the characteristics mentioned above are common to almost all LPG cases. Accordingly, it has been suspected that abnormal lipoproteins induced by apoE variants play a pivotal role in the formation of lipoprotein thrombi of LPG. Thirteen different apoE variants were detected in LPG cases since we discovered apoE Sendai (Arg145Pro) in 19974). These LPG-related apoE variants are mainly caused by point mutations of amino acid residues around the LDL-receptor binding domain, and constitute isoforms with apoE2 or E1 allele.

In this issue, cases of LPG with the same novel variant, apoE2 (Arg158Pro), are published independently, and the variants are called apoE Osaka and apoE Kurashiki, respectively ${ }^{5,6)}$. Patients in both reports were not relatives and the distance between their homes was $130 \mathrm{~km}$, although they might have had remote ancestors in common.

ApoE is an arginine-rich protein and the substitution of cysteine for arginine at position 158 is recognized as a common apoE2 mutation causing familial type III hyperlipoproteinemia ${ }^{7}$; however, the substitution of proline for arginine at the same position is not known so far. On the other hand, the substitution of proline for arginine was the first apoE mutation in LPG, called apoE Sendai (Arg145Pro) ${ }^{4}$, which comprises the majority of apoE variants related to LPG. Moreover, apoE Chicago (Arg147Pro) ${ }^{8)}$ and apoE Guangzhou (Arg150Pro) ${ }^{9)}$ have been reported in the United States and China, respectively. Proline acts as a structural disrupter in the middle of the alpha helix, and is commonly found as the first residue of the alpha helix or in turns in an amino acid arrangement ${ }^{1,4)}$. Accordingly, proline is not in or around the LDL-receptor binding domain of apoE which forms a continuous alpha helix. Once the substitution of proline occurs in this area, however, apoE may seriously change its structure, aggregate lipoproteins and induce intraglomerular lipoprotein thrombi of LPG.

It is of interest that the substitution of proline as- sociated with LPG was found at position 158, where the common apoE2 mutant inducing familial type III hyperlipoproteinemia possesses cysteine. In this point, we recognize that both reports published in this issue $^{5,6)}$ are very valuable and propose that the novel variant be called apoE Osaka/Kurashiki.

In LPG, apoE variants having 3 amino acid deletions were reported independently as apoE Tokyo (del 141-143) ${ }^{10)}$ and apoE Maebashi (del 142-144) ${ }^{11)}$. But both deletions were recognized to be the same because the amino acid arrangement was repeated between 141 and 146. Now, this variant is generally called apoE Tokyo/Maebashi and is found not only in Japan but also in China. The discovery of apoE Osakal Kurashiki will widen the scope of the study of LPG.

\section{References}

1) Saito T, Matsunaga A, Oikawa S: Impact of lipoprotein glomerulopathy on the relationship between lipids and renal diseases. Am J Kidney Dis, 2006; 47: 199-211

2) Oikawa $S$, Suzuki N, Sakuma E, Saito T, Namai K, Kotake H, Fujii Y, Toyota T: Abnormal lipoprotein and apolipoprotein pattern in lipoprotein glomerulopathy. Am J Kidney Dis, 1991; 18: 553-558

3) Saito T, Sato H, Kudo K, Oikawa S, Shibata T, Hara Y, Yoshinaga K, Sakaguchi H: Lipoprotein glomerulopathy: Glomerular lipoprotein thrombi in a patient with hyperlipoproteinemia. Am J Kidney Dis, 1989; 13: 148-153

4) Oikawa S, Matsunaga A, Saito T, Sato H, Seki T, Hoshi K, Hayasaka K, Kotake H, Midorikawa H, Sekikawa A, Hara S, Abe K, Toyota T, Jingami H, Nakamura H, Sasaki J: Apolipoprotein E Sendai (Arginine $145 \rightarrow$ Proline): A new variant associated with lipoprotein glomerulopathy. J Am Soc Nephrol, 1997; 8: 820-823

5) Mitani A, Ishigami M, Watase K, Minakata T, Yamamura T: A novel apolipoprotein E mutation, apoE Osaka (Arg158 Pro), in a dyslipidemic patient with lipoprotein glomerulopathy. J Atheroscler Thromb, 2011; 18: 531-535

6) Tokura T, Itano S, Kobayashi S, Kuwabara A, Fujimoto S, Horike H, Satoh M, Komai N, Tomita N, Matsunaga A, Saito T, Sasaki T, Kashihara N: A novel mutation apoE2 Kurashiki (R158P) in a patient with lipoprotein glomerulopathy. J Atheroscler Thromb, 2011; 18: 536-541

7) Mahley RW, Innerarity TL, Rall SC Jr, Weisgraber KH: Plasma lipoproteins: apolipoprotein structure and function. J Lipid Res, 1984; 25: 1277-1294

8) Sam R, Wu H, Yue L, Mazzone T, Schwartz MM, Arruda JA, Dunea G, Singh AK: Lipoprotein glomerulopathy: a new apolipoprotein $\mathrm{E}$ mutation with enhanced glomerular binding. Am J Kidney Dis, 2006; 47: 539-548

9) Luo B, Huang F, Liu Q, Li X, Chen W, Zhou SF, Yu X: 
Identification of apolipoprotein E Guangzhou (arginine 150 proline), a new variant associated with lipoprotein glomerulopathy. Am J Nephrol, 2008; 28: 347-353

10) Konishi K, Saruta T, Kuramochi $S$, Oikawa $S$, Saito T, Han H, Matsunaga A, Sasaki J: Association of a novel 3 -amino acid deletion mutation of apolipoprotein E (Apo E Tokyo) with lipoprotein glomerulopathy. Nephron, 1999; 83: 214-218

11) Ogawa T, Maruyama K, Hattori H, Arai H, Kondoh I, Egashira T, Watanabe T, Kobayashi Y, Morikawa A: A new variant of apolipoprotein E (apo E Maebashi) in lipoprotein glomerulopathy. Pediatr Nephrol, 2000; 14: $149-151$

\section{Takao Saito ${ }^{1}$ and Akira Matsunaga ${ }^{2}$}

${ }^{1}$ Division of Nephrology and Rheumatology, Department of Internal Medicine, Fukuoka University School of Medicine, Fukuoka, Japan

${ }^{2}$ Department of Laboratory Medicine, Fukuoka University School of Medicine, Fukuoka, Japan

Address for correspondence: Takao Saito, Division of Nephrology and Rheumatology, Department of Internal Medicine, Fukuoka University School of Medicine, 7-45-1 Nanakuma, Jonan-ku, Fukuoka 814-0180, Japan

E-mail: tsaito@fukuoka-u.ac.jp 